\title{
INTRODUÇÃO AO FÓRUM TEMÁTICO SUSTENTABBILIDADE NAS ESCOLAS DE ADMINISTRAÇÃO: TENSÕ̃S E DESAFIOS
}

Em recente evento de premiação a professores de administração que incluíram sustentabilidade no conjunto de suas disciplinas, Marina Silva se referiu à importância dos ideais identificatórios que, ao longo do tempo, vão se transformando em projetos identificatórios. O debate proposto por esta edição especial coloca em questão quais são os valores e ideários com os quais a escola de administração se identifica. Certamente sustentabilidade desponta como um deles e, que se espera, culmine na criação de uma cultura sustentável no mundo do trabalho e das organizações empresariais.

Toda sociedade cria e propaga ideias mestras que acabam guiando a forma como pensamos e atuamos no mundo, a ideia de que a empresa vive para seus resultados econômicos é uma delas. Mas a propagação desse pressuposto vem sendo histórica e paulatinamente atravessada por outra ideia mestra, a da gestão socioambientalmente responsável, configurando um cenário no qual as organizações empresariais têm de se mostrar mais permeáveis às contestações sociais próprias do capitalismo contemporâneo, antecipando-as e apresentando respostas.

As universidades e, mais especificamente, as escolas de administração não estão imunes a esse movimento, elas têm de formar um profissional mais adequado a tais desafios, uma vez que de suas salas de aula emergem gestores, líderes, profissionais que poderão prejudicar ou mitigar o desenvolvimento sustentável. Mas se a consolidação desta nova ideia mestra não ocorrerá em poucos anos, é fato que se rompeu a inércia. Preocupações e ações formativas pautadas pela lógica socioambiental podem ser observadas, como as apresentadas nesta edição especial.

Só que a tarefa não é simples, como bem discute Springett (2005), é importante estar atento para a necessidade de ajudar os estudantes a compreenderem que sustentabilidade não é somente um discurso sobre ecologia e economia, mas é essencialmente ideológica e política. Entende-se, desta forma, que educação para a sustentabilidade envolve um processo de investigação crítica que encoraje as pessoas a explorarem a complexidade e as implicações desta abordagem frente às forças econômicas, políticas, sociais, culturais, tecnológicas e ambientais que a nutrem ou a impedem. Neste sentido, o desafio das escolas de administração é 
desenvolver uma pedagogia que permita inovar, que potencialize a capacidade de encontrar caminhos alternativos aos já estabelecidos: outra forma de pensar os negócios, de negociar, de produzir, enfim. Uma aprendizagem que instigue o desenvolvimento da inteligência humana em direção à superação de modelos de empresa e de negócios cristalizados, o que significa que sua formação não pode ser apenas instrumental, mas, sobretudo, heurística (SCOTT, 2002).

Assim, a trajetória em direção à educação para sustentabilidade nas escolas de negócios é atravessada por uma série de tensões tanto de ordem macroestrutural, coletiva, como individual, que vão permear toda a proposta de mudança no projeto pedagógico, no currículo, na formação docente em administração, bem como naquilo que acontece, de fato, no interior das salas de aula.

Uma primeira tensão se refere à forma como Educação para Sustentabilidade (EpS) está sendo incorporada nas distintas instituições de ensino superior (IES) em administração, no Brasil e no mundo, se de forma impositiva ou mais consensual. Estudos recentes não têm sido muito animadores. Segundo Anderberg, Nordén e Hansson (2009), o número de casos de inserção desenhados com base em um amplo debate e participação dos docentes é muito pequeno em relação àqueles de movimento oposto, de cima para baixo. Como consequência, compromete-se o interesse e envolvimento dos docentes, estudantes e/ou profissionais que trabalham no campus com as questões da sustentabilidade (VELAZQUEZ; MUNGUIA; SANCHEZ, 2005). As escolas brasileiras de administração também apresentam essa tendência. Um estudo, realizado por Carvalho (20II), com coordenadores de curso de I7 IES do Brasil mostra que o cenário aqui é o mesmo que o de países estrangeiros. Raras são as experiências em que o corpo docente fez parte das decisões em relação aos processos de mudança curricular ou na gestão das próprias IES.

Soma-se a essa questão o papel que os coordenadores de curso de administração, diretores, reitores, enfim, a liderança acadêmica tem exercido neste contexto, ora facilitando, ora dificultando mudanças. Quais instrumentos de incentivo e mecanismos institucionais lançam mão de ações para o fomento à EpS, em termos de bolsas de estudo e projetos de pesquisa na área nas IES, públicas e privadas? Como valorizam (ou não) docentes que inovam em suas disciplinas de finanças, estratégia, gestão de pessoas, e colocam na sua agenda de ensino questões de sustentabilidade?

Bruno e Hessel (20II) destacam que, historicamente, a administração escolar é entendida como uma função especializada, verticalmente hierarquizada e autoritária. O gestor educacional acaba apoiando-se em uma estrutura piramidal de poder e se distancia daqueles que devem se constituir seus parceiros de trabalho mais importantes, ou seja, os docentes. Assim, é possível pensar que os gestores acadêmicos 
[...] precisam perceber o caráter transformador de sua ação educativa e reconhecer a escola como um elemento vivo, rico em relações interpessoais que podem ser valorizadas no processo de idealização e concretização do saber pedagógico. Além disso, precisam compreender a cultura e clima da sua escola para estimular a interação entre os elementos da equipe de educadores, a fim de promover a construção de uma identidade grupal e a execução das mudanças estruturais necessárias a um processo auto-organizativo (BRUNO; HESSEL, 20II, p. 87-88).

Sem essas mudanças na postura do gestor educacional, não há como pensar em criar mecanismos que tornem projetos pedagógicos com ênfase em sustentabilidade em ações efetivamente coletivas no âmbito das IES. Um gestor comprometido com um projeto coletivo de universidade sustentável favorece a construção de projetos interdisciplinares e incentiva a contratação de docentes com perfis diferenciados, em consonância com as preocupações socioambientais.

Este cenário conduz à segunda tensão que vivem as IES que se propõem a incorporar uma filosofia de educação sustentável. As escolas de administração tradicionalmente apresentam um projeto pedagógico e uma estrutura curricular distante da lógica interdisciplinar e/ou transdisciplinar que se defende na formação para sustentabilidade. Como bem expressa Springett (2005), em nenhum outro curso o desafio que a insustentabilidade ecológica e social impõe para o currículo formal revelou-se mais problemático do que nos currículos dos cursos de gestão das escolas de negócios, frente ao grau de oposição encontrado por educadores que tentam desenvolver narrativas alternativas ou abordagens interdisciplinares. Embora propostas multidisciplinares, muitas vezes, possam fornecer lentes que desenvolvam nos alunos habilidades de questionamento, fazendo-os repensar a ideologia dominante que direciona os cursos de gestão de negócios (BANERJEE, 2004), elas ainda provocam resistências.

Embora tais propostas sejam incentivadas no documento que propõe a Década da Educação das Nações Unidas para o Desenvolvimento Sustentável, com duração de 2005 a 2014 (UNESCO, 2005), ainda se observa poucas oportunidades objetivas para o diálogo interdisciplinar entre os docentes. Um aspecto comentado por Springett e Kearins (200I) é que apesar do aumento da variedade de cursos oferecidos no ensino superior, com novas opções de graduação instituídas, os estudantes continuam a ter as mesmas restrições ao número total de disciplinas de que podem participar, limitando a transversalidade e a possibilidade do aluno buscar outros campos do saber, como advoga a sustentabilidade. Embora o desejo por uma inter/transdisciplinaridade esteja claramente manifestado em discursos de alguns coordenadores de curso de administração no Brasil, questiona-se se ela já é uma realidade nas IES. Os depoimentos dos entrevistados por 
Carvalho (20II) indicam que iniciativas desta natureza se constituem muito mais em um ideal a ser perseguido do que uma realidade.

Se a inserção da sustentabilidade com base na integração de uma disciplina específica no ensino é um alvo comum de críticas (TILBURY, 2004; STERLING, 200I), a formação de profissionais com perfil multidisciplinar para atuarem nos programas torna-se prioridade (RUPEA, 2005), o que indica uma terceira tensão relativa aos processos de formação do professor para trabalhar com as questões de sustentabilidade e o desenvolvimento de estratégias de ensino-aprendizagem condizentes com essa natureza de conteúdo.

A postura tradicional do professor tem se constituído em um aspecto que dificulta a sua forma de olhar para questões de sustentabilidade nas escolas de negócios, como tem sido destacado por diferentes autores nacionais (TELLES, 2OII; CARVALHO, 2OII; GONÇALVEZ-DIAS; BELOQUE; HERRERA, 2OII; TOZONI-REIS, 200I) e internacionais (SPRINGETT, 2005; ELLIOTT, 2004; FIEN; MACLEAN, 2000). Embora tais autores tratem a questão sob perspectivas distintas, há elementos comuns que se pode destacar. Elliott (2004), por exemplo, ressaltam que a educação gerencial é normalmente atomizada, constituída de silos, mais focada no conhecimento especializado (tais como marketing, recursos humanos) do que no conhecimento de grandes áreas. Assim, apenas a introdução de disciplinas ligadas à sustentabilidade, isoladamente, perpetua a tendência à fragmentação do conhecimento e o seu tratamento tangencial, como enfatiza Springett (2005).

Há ainda que se considerar a maneira como os professores ensinam os conteúdos de tais disciplinas na sala de aula. Um estudo realizado por Down (2006) em uma universidade da Jamaica destaca que um dos principais desafios enfrentados pela universidade está relacionado ao conhecimento, entendimento e atitude dos professores sobre o tema, que varia conforme o grau de engajamento ou ceticismo do corpo docente. Os docentes se questionam sobre a própria conceituação da sustentabilidade e sobre a maneira de tratar tal conteúdo no âmbito das suas disciplinas. Para lidar com essa situação, o autor relata uma experiência em que se buscou promover na universidade um aprofundamento na literatura sobre EpS, fazendo os docentes refletirem sobre o tema, tomando como referência básica os documentos da Unesco. Foi a maneira que encontraram para os professores se conscientizarem a respeito da forma fragmentada com que trabalhavam, passando a incorporar a ideia de que qualquer proposta de EpS envolve uma abordagem holística e interdisciplinar.

Ainda em relação às questões de inter e transdisciplinaridade, alguns documentos de organismos internacionais procuram fornecer pistas para uma reflexão sobre essa tensão. Dentre eles, a United Nation Economic Comission for Europe (UNECE) - publicou, em 2008, os resultados de uma ampla discussão sobre as 
deficiências existentes no processo de formação de professores em todos os níveis, desde a pré-escola até o ensino universitário. O documento, produzido com a participação de I5 universidades de diferentes países (Áustria, Alemanha, Espanha, Bélgica, Suíça, Dinamarca e Reino Unido) foi denominado Competencies for ESD (Education for Sustainable Development) teachers (SLEURS, 2008). Nele é apresentado um conjunto de competências que deveriam orientar as propostas de formação dos professores para ensinar sustentabilidade. Dentre elas, destaca-se a importância que tais professores utilizassem metodologias inovadoras de ensino que possibilitassem aos alunos trabalharem holisticamente sobre os problemas relacionados ao desenvolvimento sustentável, promovendo o relacionamento entre a teoria e a prática. As metodologias também deveriam ajudar os estudantes a lidar com as incertezas, ambiguidades e complexidades que permeiam o seu entorno em relação às questões de sustentabilidade, procurando fazê-los não só compreender suas raízes e especificidades, mas também apresentar possíveis soluções de forma sistêmica. Além disso, o documento instiga os professores a serem capazes de analisar as questões de poder local, lidar com situações políticas, pensar e agir estrategicamente, assim como facilitar redes de contato, a fim de construírem conhecimentos relevantes para a EpS.

Vale ainda fazer referência ao Centro de Pesquisa Aries (Australian Research Institute for Environment and Sustainability), localizado na Universidade Macquarie, em Sydney, Austrália. Criado em 2003 com base em uma iniciativa do próprio governo australiano para a realização de projetos entre governo, comunidade e organizações empresariais, o Centro tem a intenção de desenvolver a capacidade desses atores sociais em alcançar melhorias em questões que envolvem sustentabilidade. A proposta se configura um forte apelo colaborativo para uma mudança nos padrões de gestão, no engajamento de stakeholders e no desenvolvimento organizacional. Consta das publicações do instituto ARIES que uma das principais dificuldades da EpS em escolas de negócios da Austrália é a falta de professores com experiência e conhecimento em sustentabilidade. Para minimizar o problema, desenvolveram um conjunto de recursos para auxiliar docentes do ensino superior a ensinarem sustentabilidade.

De forma análoga, no Brasil, Tozoni-Reis já em 200I fez uma crítica à formação fragmentada de docentes para sustentabilidade, tendo em vista pensar no desenho de um possível projeto de formação interdisciplinar. No caso de Telles (20II), Carvalho (20II) e Gonçalves-Dias, Beloque e Herrera (20II), a crítica se concentra na dificuldade que as escolas de negócios vêm enfrentando para compor um corpo docente qualificado para tratar a temática. A pesquisa de Carvalho (20II) com coordenadores de curso mostra que os programas de formação docente com este foco têm se constituído uma discussão acessória nas universidades brasileiras e estão distantes da necessidade de repensar o perfil dos administradores formados. 
Por fim, vinculada ainda à questão da formação, há a dificuldade de os docentes proporem estratégias didático-pedagógicas que atendam ao que a literatura na área considera importante no ensino de sustentabilidade, como o trabalho com simulações, estudos de caso, técnicas de resolução de problemas, entre outras (KEARINS; SPRINGETT, 2003; SVOBODA; WHALEN, 2004; ELLIOTT, 2004; ANANDALE; MORRISON-SAUNDERS, 2004; SPRINGETT, 2005; COLLINS; KEARINS, 2007; GONÇALVES-DIAS; BELOQUE; HERRERA, 2OII).

Há ainda uma quarta tensão que merece ser mencionada, trata-se da ruptura entre a ênfase dada aos aspectos relacionados à gestão sustentável do campus e àqueles que se dirigem ao envolvimento dos indivíduos nas instituições universitárias. De um lado, o fato de que as próprias IES ainda não conseguiram incorporar, em sua dinâmica organizacional, os princípios e práticas sustentáveis que pretendem ensinar, gera um descrédito interno entre alunos, professores e funcionários (TAUCHEN; BRANDLI, 2006; KRAEMER, 2006). Por outro lado, também é possível observar um movimento oposto, com iniciativas focadas na gestão operacional do campus (redução da pegada ecológica; minimização de impactos ambientais e sociais causado pela gestão operacional do campus; ações em prol da acessibilidade; política de compras sustentáveis; economia de energia; redução de resíduos), sem que nenhum redesenho significativo ou mudança curricular profunda esteja em curso (CALDER; CLUGSTON, 2003).

Todas essas tensões são, ao mesmo tempo, desafios a serem enfrentados. Desafios que já vêm sendo objeto de atenção por parte de distintos atores sociais no Brasil, sejam eles professores, pesquisadores, escolas superiores de administração, universidades, ou mesmo a própria Capes, como se pode ver na descrição das experiências publicadas nesta edição, apresentadas a seguir.

Há que se destacar que o Fórum Temático da RAM representa a continuidade do esforço de um grupo de professores-pesquisadores em desenvolver ações de ensino, pesquisa e extensão que contribuam com o objetivo de promover a EpS por meio de um projeto - Pró-Administração - financiado pela Capes, e que se rege por uma característica interdisciplinar e de solidariedade interprogramas visando a capacitação e formação de recursos humanos para a educação em administração. Para alcançar esse objetivo o projeto vem desenvolvendo um conjunto de ações, articuladas entre seus parceiros e com outras comunidades de aprendizagem, preocupadas com a questão da inserção da temática da sustentabilidade no âmbito dos cursos de administração de empresa, sempre promovendo um processo dialógico de comunicação e disseminação de conhecimentos entre pesquisadores, professores e alunos de graduação e pós-graduação.

Assim, a chamada que se fez para organizar este Fórum Temático procurou instigar professores e pesquisadores a descrever, analisar e refletir sobre o espaço 
formativo que a universidade promove por meio de suas lideranças acadêmicas, visando a formação de administradores para a sustentabilidade, discutindo e revendo seus projetos pedagógicos e currículos, desenvolvendo experiências didático-pedagógicas no âmbito da EpS, envolvendo e ouvindo seus alunos e professores como atores sociais fundamentais desse esforço.

Os artigos aprovados e que constam deste Fórum revelam o esforço dos autores em promover a reflexão a respeito da temática, que aqui é apresentada com base em enfoques diversos. Acredita-se que os artigos ofereçam oportunidades para os leitores se engajarem em um processo de reflexão crítica, e que, com base nos conteúdos ali apresentados e desenvolvidos, estabeleçam um profícuo diálogo individual (do leitor com seus pressupostos e experiências internas) e coletivo (do leitor como ser social capaz de reconhecer a natureza social, histórica e política das ideias e experiências relatadas).

Inicia-se a apresentação dos artigos com o ensaio teórico de Venzke e Nascimento, que discute "a necessidade de geração de novos arranjos nos conhecimentos para a inserção, de forma mais ampla e complexa, da sustentabilidade socioambiental como caminho para a definição de um marco conceitual na formação do administrador". O artigo parte de uma discussão acerca das bases epistemológicas que orientam os conteúdos tratados nos cursos de administração no Brasil para, a seguir, analisar o que se entende por sustentabilidade socioambiental e a natureza do conhecimento produzido sobre o tema. A partir daí, propõe a ideia dos arranjos do conhecimento como um possível caminho para se pensar a inserção da sustentabilidade socioambiental na formação dos administradores, recorrendo então às proposições do pensamento complexo para embasar os argumentos desenvolvidos. Ao final do artigo os autores convidam os leitores a continuarem a refletir sobre o tema, buscando "com os diferentes atores, que pensam e executam o ensino e a pesquisa em administração, percepções e conhecimentos sobre a construção do caminho em direção à inserção da sustentabilidade socioambiental na formação dos administradores brasileiros, de forma plural e reflexiva".

Acredita-se que o exame de aspectos relativos ao ensino e pesquisa em gestão ambiental nos programas brasileiros de pós-graduação em administração, feito por Sinay, Dalbem, Loureiro e Vieira, também se constitui importante contribuição, uma vez que ressalta "o papel fundamental da universidade como catalisadora para que a sociedade coloque em prática os princípios da sustentabilidade". Partindo do atual estágio da legislação brasileira a respeito da educação ambiental, o segundo artigo desta edição especial apresenta os resultados de uma pesquisa documental que conclui que o ensino e a pesquisa da pós-graduação em administração no Brasil "ainda não possui a expressividade desejada sobre tema tão importante quanto o da gestão ambiental”. Os autores destacam 
também a importância da produção científica na área ressaltando que ela depende da capacitação de professores e estudantes. Nesse sentido, chamam atenção para "a necessidade de incluir nas grades curriculares da pós-graduação disciplinas e/ou tópicos que colaborem com a formação de profissionais capacitados não apenas para esta produção, mas também para administrar organizações contemporâneas de todos os setores".

A experiência de inserção da EpS em instituições de ensino e nos cursos de gestão está presente em dois artigos desta edição especial.

O artigo de Palma, Alves e Silva examinou como questões relacionadas a EpS estão sendo tratadas nos institutos federais brasileiros, com destaque para o Instituto Federal de Educação, Ciência e Tecnologia do Rio Grande do Sul (IFRG). Traz os resultados alcançados em uma pesquisa de caráter exploratório. Inicialmente verificou-se "se esse tema tem sido abordado nos Projetos Pedagógicos dos Cursos (PPCs) do IFRS relacionados ao eixo tecnológico Gestão e Negócios". Além disso, foi realizada coleta com professores e coordenadores dos cursos "para verificar como o assunto tem sido abordado nas disciplinas e nas políticas dos campi do IFRG". Em complemento "são apresentados brevemente dois projetos de cursos, dos campi de Canoas e Osório, que têm como foco a sustentabilidade". Os resultados mostram que o IFRG apresenta preocupação com o assunto em seus documentos, mas que não existe uma política institucional que trate das ações relativas à sustentabilidade na organização. A introdução do tema nos cursos ocorre a partir da iniciativa dos docentes, embora projetos na área já existam, como demonstrado pelas iniciativas presentes nos campi de Canoas e Osório. Ao concluírem o artigo, os autores comentam que, se as escolas realmente seguirem as orientações da Unesco (2005) e os institutos federais "colocarem em prática o que se propõem em seus documentos - verticalização do ensino (atuando em diferentes níveis e modalidades), integração e desenvolvimento do tripé ensino, pesquisa e extensão - eles poderão tornar-se instituições propícias para o desenvolvimento de pesquisas a ações na direção da ESD".

Já o artigo de Gonçalves-Dias, Herrera e Cruz analisa os desafios e dilemas enfrentados para a inserção da disciplina por uma instituição de ensino superior localizada na cidade de São Paulo, "Sustentabilidade" no curso de administração. O estudo de caso desenvolvido, de natureza exploratória, adotou uma metodologia mista. Os dados foram analisados de maneira qualitativa e quantitativa, e a construção do artigo está baseada na pesquisa documental, na realização de entrevistas e na própria vivência dos autores como docentes do curso de administração. O estudo mostra que as ações desenvolvidas pela universidade estudada representaram uma mudança significativa para o curso de administração, no qual se incluiu a disciplina "Sustentabilidade". Embora tenha sido detectado o esforço institucional na introdução do tema nos diálogos, na agenda de interesse 
e nos processos formativos, os autores alertam que "permanece o risco de que uma disciplina isolada em um programa dominado pelo paradigma econômico apresente-se como medida imediatista que atende a demanda por sustentabilidade puxada pela lógica de mercado". O texto comenta e analisa os desafios enfrentados por este tipo de iniciativa, conforme comentado por outros autores no início deste texto.

A preocupação em detectar as opiniões e percepções dos alunos em relação à relevância da sustentabilidade nos conteúdos trabalhados no currículo e em disciplinas do curso de administração constitui o foco dos próximos dois artigos que constam desta edição especial.

O artigo apresentado por Silva, Czykiel, Figueiró, Santos e Galvão teve como objetivo verificar "como a EpS pode contribuir para o processo de tomada de decisão consciente" de discentes do curso de administração da cidade de Recife. A pesquisa adotou uma abordagem quantitativa e utilizou um questionário para coleta de dados. Com base nos resultados obtidos, os autores consideram que a identificação dos alunos quanto ao consumo consciente torna possível a realização de "uma discussão sobre como a EpS pode interferir e contribuir para a construção de uma visão mais consciente" por parte dos mesmos.

A pesquisa apresentada por Costa, Ramos e Mazza também apresenta um novo e interessante "olhar" que procura responder a seguinte questão de pesquisa: "como os valores pessoais se associam às atitudes dos estudantes de administração em relação à área de gestão socioambiental?” buscando componentes explicativos "que estão além do alcance direto do ambiente de formação". O estudo quantitativo examinou "as avaliações e intenções futuras de envolvimento dos estudantes dos cursos de administração com a área socioambiental e os condicionantes oriundos de valores pessoais associados à relação entre humanos e natureza". Os resultados indicam uma "relação negativa do conservadorismo antropocêntrico com a avaliação pelos estudantes do curso de administração da importância da gestão socioambiental no curso, e com seu interesse de envolvimento com a área no futuro". Além disso, mostram que "em relação à percepção de domínio humano sobre a natureza que, embora pareça estar sofrendo alterações ao longo dos anos, essa mudança não influencia o interesse geral dos alunos pela área socioambiental".

Considera-se que o conjunto da produção que compõe este Fórum Temático traz contribuições relevantes às tensões e desafios apresentados na primeira parte desta apresentação. No entanto, a falta de estudos que apresentem a visão dos professores como importante ator social na implementação e concretização dos projetos pedagógicos chamam nossa atenção, e se constitui em uma lacuna que oferece oportunidades para a realização de futuras pesquisas.

Com esta edição especial criamos novas oportunidades de diálogos, tomando contato com professores e/ou pesquisadores envolvidos com a EpS que colabora- 
ram conosco nesta tarefa de levar à comunidade acadêmica estudos teóricos e pesquisas empíricas capazes de instigar nossa curiosidade e aprofundar nossa compreensão acerca do tema.

\section{ARILDA SCHMIDT GODOY}

Editora convidada

Doutora em Educação pela Universidade de São Paulo (USP).

Professora do Programa de Pós-Graduação em Administração

da Universidade Presbiteriana Mackenzie (UPM).

Rua Consolação, 896, Consolação, São Paulo - SP - Brasil - CEP 01302-907

E-mail: arilda.godoy@mackenzie.br

JANETTE BRUNSTEIN

Editora convidada

Doutora em Educação pela Universidade de São Paulo (USP).

Professora do Programa de Pós-Graduação em Administração

da Universidade Presbiteriana Mackenzie (UPM).

Rua Consolação, 896, Consolação, São Paulo - SP - Brasil - CEP 01302-907

E-mail: janette.gorodscy@mackenzie.br

\section{TÂNIA MARIA DIEDERICHS FISCHER}

Editora convidada

Doutora em Administração pela Faculdade de Economia, Administração

e Contabilidade da Universidade de São Paulo (FEA-USP).

Professora do Programa de Pós-Graduação em Administração da Universidade Federal da Bahia (UFBA). Avenida Reitor Miguel Calmon, s/n, $3^{\circ}$ andar, Vale do Canela, Salvador - BA - Brasil - CEP 40110-903 E-mail: taniafischer@ciags.org.br

\section{REFERÊNCIAS}

ANDERBERG, E.; NORDÉN, B.; HANSSON, B. Global learning for sustainable development in higher education: recent trends and a critique. International Journal of Sustainability in Higher Education, v. IO, n. 4, p. 368-378, 2009 .

ANNANDALE, D.; MORRISON-SAUNDERS, A. Teaching process sustainability: a role-play case focused on finding new solutions to a waste-water management problem. In: GALEA, C. Teaching business sustainability: cases, simulations and experimental Sheffield: Greenleaf Publishing Limited, 2004. p. I80-I98. 2. v. 
BANERJEE, S. B. Teaching sustainability: a critical perspective. In: GALEA, C. Teaching business sustainability: volume I from theory to practice. Sheffield: Greenleaf Publishing Limited, 2004. p. 34-47. I v.

BRUNO, A. R.; HESSEL, A. M. di G. Implicações das tecnologias da informação e da comunicação para a educação ambiental: formando comunidades colaborativas de gestores educacionais. In: Educação para a era da sustentabilidade. São Paulo: Saint Paul, 20II. p. 85-97.

CALDER, W.; CLUGSTON, R. Progress toward sustainability in higher education. Washington: Environmental Law Institute, 2003.

CARVALHO, S. L. G. Educação para sustentabilidade em escolas de administração de empresas: a perspectiva de coordenadores acadêmicos no Brasil. Dissertação (Mestrado em Administração de Empresas)-Universidade Presbiteriana Mackenzie, São Paulo, 20II.

COLLINS, E.; KEARINS, K. Exposing students to the potential and risks of stakeholder engagement when teaching sustainability: a classroom exercise. Journal of Management Education, v. 3I, n. 4, p. 52I-540, 2007.

DOWN, L. Addressing the challenges of mainstreaming education for sustainable development in higher education. International Journal of Sustainability in Higher Education, v. 7, n. 4, p. 390-399, 2006. ELLIOTT, S. R. Approaching sustainability through a business-science synthesis. In: GALEA, C. Teaching Business Sustainability: from theory to practice. Sheffield: Greenleaf Publishing Limited, 2004. P. I5I-I55. V. I.

FIEN, J.; MACLEAN, R. Teacher education for sustainability: Two teacher education projects from Asia and the Pacific. Journal of Science Education and Technology, v. 9, n. I, p. 37-48, 2000.

GONÇALVES-DIAS, S. L. F.; BELLOQUE, M. C. M.; HERRERA, C. B. Desafios para inserção da disciplina "Sustentabilidade" em cursos de Administração: a experiência de uma Instituição de Ensino Superior paulistana. In: ENSINO E PESQUISA EM ADMINISTRAÇÃO, XIV., 20II, São Paulo. Anais... São Paulo: SEMEAD, 20II. p. I-I7.

KEARINS, K.; SPRINGETT, D. Educating for sustainability: developing critical skills. Journal of Management Education, v. 27, n. 2, p. I88-204, 2003.

SCOTT, W. Education and sustainable development: challenges, responsibilities, and frames of mind. The Trumpeter, v. I8, n. I, p. 22-34, 2002.

Seminário Mapeamento da educação ambiental em instituições brasileiras de educação superior: elementos para discussão sobre políticas públicas. [S.l.: s. n.], RUPEA, 2005.

SLEURS, W. Competencies for ESD (Education for Sustainable Development) teachers: A framework to integrate ESD in the curriculum of teacher training institutes, 2008. Disponível em: <http://www. unece.org>. Acesso em: 20 jan. 2013.

SPRINGETT, D.; KEARINS, K. Gaining legitimacy? Sustainable development in business school curricula. Sustainable Development. v. 9, n. 4, p. 213-22I, $200 \mathrm{I}$.

SPRINGETT, D. Education for sustainability in the business studies curriculum: a call for a critical agenda. Business Strategy and the Environment, v. I4, n. 3, p. I46-I59, 2005.

STERLING, S. Sustainable education: re-visioning learning and change. Dartington: Green Books, 200 I. SVOBODA, S.; WHALEN, J. Using experiential simulation to teach sustainability. In: GALEA, C. Teaching business sustainability: cases, simulations and experimental approaches. Sheffield: Greenleaf Publishing Limited, 2004. p. I7I-I79. 2. v. 
TAUCHEN, J.; BRANDLI, L. L. A gestão ambiental em instituições de ensino superior: modelo para implantação em campus universitário. Gestão \& Produção, v. I3, n. 3, p. 503-515, set.-dez. 2006. TELLES, B. M. Integrando a sustentabilidade na formação de administradores. São Paulo: Pontifícia Universidade Católica de São Paulo, 20ıı.

TILBURY, D. No title environmental education for sustainability: a force for change in higher education. In: CORCORAN, P. B.; WALS, A. E. J. (Ed.). Higher education and the challenge of sustainability: problematics, promise and practice. Dordrecht: Kluwer Academic Publishers, 2004. p. 97-II2. TOZONI-REIS, M. F. C. Educação ambiental: referências teóricas no ensino superior. Interface Comunic., Saúde, Educ., v. 5, n. 9, p. 33-50, 200 I.

VELAZQUEZ, L.; MUNGUIA, N.; SANCHEZ, M. Deterring sustainability in higher education institutions: An appraisal of the factors which influence sustainability in higher education institutions. International Journal of Sustainability in Higher Education, v. 6, n. 4, p. 383-39I, 2005.

UNESCO. Década da Educação das Nações Unidas para um desenvolvimento sustentável, 2005-2014: documento final do esquema internacional de implementação. Brasília, 2005. Disponível em: $<$ http://unesdoc.unesco.org/images/0013/001399/>. Acesso em: jan. 2013. 